\title{
Esplenectomía laparoscópica en paciente con síndrome de Evans
}

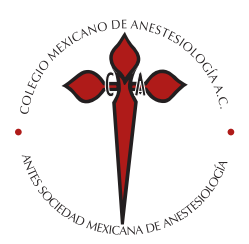

\author{
Laparoscopic splenectomy in patient with \\ Evans syndrome
}

\author{
Dr. José Eduardo Bendaña,* Dra. Lea Crisel Salinas-Vallecillo ${ }^{\ddagger}$
}

Citar como: Bendaña JE, Salinas-Vallecillo LC. Esplenectomía laparoscópica en paciente con síndrome de Evans. Rev Mex Anestesiol. 2022; 45 (2): 135-137. https://dx.doi.org/10.35366/103889

\begin{abstract}
RESUMEN. El síndrome de Evans es una entidad rara que se presenta aproximadamente en 3.6 por cada millón de habitantes; siendo más común en el sexo femenino. Éste se caracteriza por la presencia de anemia hemolítica autoinmunitaria idiopática y púrpura trombocitopénica idiopática. Se presenta el caso de paciente femenino de 42 años de edad sin mejora en su nivel de plaquetas, por lo que se decide realizar esplenectomía mediante laparoscopía. La paciente, previo a la intervención quirúrgica, presenta nivel de plaquetas consideradas límites, 53,000/ $\mathrm{L}$, por lo que se decide transfundir plaquetas previo al procedimiento. Durante la evaluación preanestésica se consignaron múltiples factores de riesgo para considerar una vía aérea difícil. Se optimizó el nivel de plaquetas y se mantuvieron esteroides perioperatorios. Se decidió dar anestesia general endotraqueal, con resultados satisfactorios durante el procedimiento quirúrgico.
\end{abstract}

ABSTRACT. Evans syndrome is a rare syndrome that occurs in approximately 3.6 per million inhabitants; being more common in women. Its characteristics are the presence of idiopathic autoimmune hemolytic anemia and idiopathic thrombocytopenic purpura. We present a case of a 42-year-old patient with no improvement in her platelet level, so it was programmed to perform a laparoscopic splenectomy. The patient before the surgical intervention presented a level of platelets considered limit, $53,000 / \mu \mathrm{L}$, so it was decided to transfuse platelets before surgery. During the pre-anesthesia evaluation, multiple risk factors were recorded to be considered as a difficult airways. Thrombocytopenia was improved and perioperative steroids were maintained. It was decided to administer general endotracheal anesthesia, obtaining satisfactory anesthesia during the surgical procedure.

\begin{abstract}
Palabras clave: Síndrome de Evans, esplenectomía, trombocitopenia.
\end{abstract}

Keywords:

Evans syndrome, splenectomy, thrombocytopenia.

\author{
* Residente de Postgrado en \\ Anestesiología, Reanimación \\ y Dolor, Universidad Nacional \\ Autónoma de Honduras, \\ Tegucigalpa. Honduras. \\ ‡ Médico Especialista en \\ Anestesiología, Reanimación \\ y Dolor, Hospital Escuela, \\ Tegucigalpa. Honduras. \\ Correspondencia: \\ Dr. José Eduardo Bendaña \\ E-mail: jbendana92@gmail.com
}

Recibido: $14-07-2020$

Aceptado: 09-10-2020

\section{INTRODUCCIÓN}

$\mathrm{E}$ l síndrome de Evans fue descrito en 1951 por Robert Evans. Éste es una combinación de anemia hemolítica autoinmunitaria idiopática y la presencia de púrpura trombocitopénica idiopática. Este síndrome raro tiene prevalencia de 3.6 por cada millón de personas, el cual tiene una mortalidad elevada de $30 \%$ a un año del diagnóstico. Es más común en el sexo femenino con aproximadamente $60-70 \%$ de los pacientes $^{(1)}$.

El diagnóstico del síndrome de Evans se realiza por exclusión durante el examen físico, en el cual se obtendrán características clínicas de anemia, trombocitopenia o ambas, como pueden ser palidez, ictericia, equimosis, epistaxis y, debido al consumo de terapia inmunosupresora, la presencia de infecciones recurrentes. En los exámenes de laboratorio es común encontrar anemia, trombocitopenia, esferocitosis, aumento de bilirrubina y aumento de lactato deshidrogenasa; también pueden presentarse anticuerpos antiplaquetarios positivos. Esta enfermedad crónica se caracteriza por períodos de remisión y exacerbaciones ${ }^{(2,3)}$.

No existe tratamiento definitivo para el síndrome de Evans ni cuándo iniciarlo, éste dependerá de la experiencia del médico tratante y de la situación clínica específica del paciente. Entre los tratamientos de primera línea están los esteroides e inmunoglobulina hiperinmune intravenosa. La esplenectomía puede ser útil en el tratamiento cuando éste no responde de manera satisfactoria a la farmacoterapia, o con el objetivo de disminuir recaídas o el uso de esteroides. La esplenectomía presenta una alta tasa de complicaciones, como puede ser: sangrado, infecciones por microorganismos encapsulados y eventos tromboembólicos. Debido a estas complicaciones es que la esplenectomía está siendo reemplazada por el rituxi$\mathrm{mab}^{(3-5)}$. 


\section{CASO CLÍNICO}

Paciente femenino de 42 años de edad, con síndrome de Evans diagnosticado hace un año; tratada con azatioprina $50 \mathrm{mg}$ cada ocho horas y prednisona $12.5 \mathrm{mg}$ cada 12 horas. Antecedente de asma tratada de manera sintomática con inhaladores de rescate (salbutamol dos inhalaciones de $100 \mu \mathrm{g}$ cada una), última crisis presentada hace tres años. Presenta depresión diagnosticada hace tres meses tratada con amitriptilina $25 \mathrm{mg}$ cada día y fluoxetina $20 \mathrm{mg}$ cada día. Presenta ultrasonido que refiere bazo de forma y ecogenicidad normal con tamaño de $10.84 \times 4$.9. Fue tratada en múltiples ocasiones con inmunoglobulinas intravenosas sin respuesta, al momento de la evaluación presenta trombocitopenia de $53,000 / \mu \mathrm{L}$, eritrocitos de $397,000 / \mu \mathrm{L}$, hemoglobina de $12.0 \mathrm{~g} / \mathrm{dL}$, glucosa de $87 \mathrm{mg} / \mathrm{dL}$, creatinina $0.63 \mathrm{mg} / \mathrm{dL}$, bilirrubina total de 0.8 $\mathrm{mg} / \mathrm{dL}$, lactato deshidrogenasa $253 \mathrm{U} / \mathrm{L}$, índice internacional normalizado (INR, por sus siglas en inglés) de 0.9 , por lo que se decide realizar esplenectomía laparoscópica programada. Presenta antecedentes quirúrgicos de dos cesáreas previas, la primera bajo anestesia neuroaxial sin complicaciones, la segunda presentó sufrimiento fetal agudo, por lo que se decidió administrar anestesia general y presentó broncoaspiración como complicación, paciente resolvió satisfactoriamente. Asimismo, señala dos antecedentes de hospitalización por trombocitopenia menor a $50,000 / \mu \mathrm{L}$.

A la exploración física: peso de 68 kg, altura de 1.52 metros, índice de masa corporal de 29.4, mucosas y tegumentos ligeramente pálidos. Presencia de equimosis en ambos brazos secundarias a canalizaciones intravenosas no satisfactorias. Ritmos cardíacos rítmicos, regulares. A la exploración respiratoria entrada de aire simétrica, expansibilidad torácica sin alteraciones, no se auscultan ruidos patológicos. A la exploración de la vía aérea se puede observar Mallampati III, apertura oral $3 \mathrm{~cm}$, Patil-Aldreti grado III, Bellhouse-Dore grado II y protrusión dental grado II. Equivalentes metabólicos 5. Se pidió optimizar plaquetas previo a cirugía, por lo que se administraron 20 unidades de plaquetas. Previo a la cirugía la paciente presentaba $278,000 / \mu \mathrm{L}$ de plaquetas. Se premédica con ranitidina $50 \mathrm{mg}$, hidrocortisona $150 \mathrm{mg}$ y metoclopramida $10 \mathrm{mg}$.

Se monitoriza con electrocardiograma continuo en derivaciones II y V5, presión arterial no invasiva y oximetría de pulso. Signos vitales basales de: presión arterial 138/87 mmHg, frecuencia cardíaca de 57 latidos por minuto y oximetría de 99\%. Posteriormente, se realiza inducción anestésica con midazolam $2 \mathrm{mg}$, fentanilo $200 \mu \mathrm{g}$, propofol $150 \mathrm{mg}$, rocuronio $40 \mathrm{mg}$ y se procede a realizar intubación endotraqual con tubo 7.0 en el primer intento sin complicaciones. Se acopla a ventilación mecánica bajo los siguientes parámetros: volumen tidal $450 \mathrm{~mL}$, frecuencia respiratoria 12 por minuto, presión positiva al final de la espiración (PEEP, por sus siglas en inglés) de $5 \mathrm{~cm}$, relación inspiración-expiración de 1:2, flujo de oxígeno a 2 litros/minuto bajo modalidad controlado por volumen. Mantenimiento con sevofluorano a $2-2.5 / \mathrm{Vol} \%$, fentanilo tres dosis de $50 \mu \mathrm{g}$ y dosis adicional de $10 \mathrm{mg}$ de rocuronio. Período perioperatorio estable; se administró como coadyuvante dexketoprofeno $50 \mathrm{mg}$, dexametasona $8 \mathrm{mg}$ y morfina $7 \mathrm{mg}$. Balance hídrico menos de $130 \mathrm{~mL}$, sangrado aproximado de $120 \mathrm{~mL}$ sin necesidad de transfusión, se tenían preparadas tres unidades de glóbulos rojos empacados, tres unidades de plaquetas y dos unidades de plasma fresco congelado.

Termina procedimiento sin eventos críticos anestésicos o quirúrgicos; la paciente pasa a sala de recuperación postanestésica, extubada, respirando espontáneamente con escala de Aldrete de 9/10, escala visual análoga del dolor 0/10, con signos vitales: frecuencia respiratoria 14, presión arterial 110/68, frecuencia cardíaca 65 latidos por minuto y saturación de oxígeno de $99 \%$.

\section{DISCUSIÓN}

El objetivo de la esplenectomía en este tipo de pacientes es mejorar o estabilizar el conteo plaquetario. En una serie de cinco casos con síndrome de Evans se demostró que la cirugía era segura. El posterior seguimiento de estos casos resultó en dos pacientes que remitieron, con plaquetas mayor a 100,000/ $\mu \mathrm{L}, \mathrm{y}$ un paciente que presentó breve mejoría y posteriormente necesitó acompañamiento con terapia farmacológica ${ }^{(6)}$.

Debido al riesgo de presentar infecciones por microorganismos encapsulados, se considera oportuno la verificación o aplicación de vacunas contra Streptococcus pneumoniae, Neisseria meningitidis y Haemophilus influenzae de 15 días a seis semanas previo a la cirugía. En este caso, la aplicación de estas vacunas no fue verificada. La importancia de la correcta aplicación de vacunas así como la necesidad del uso de antibióticos de manera profiláctica posterior a la cirugía pueden disminuir el riesgo de infección postesplenectomía, la cual es más común durante los primeros dos años y puede alcanzar hasta el $50 \%$ de mortalidad, principalmente si es por Streptococcus pneumoniae ${ }^{(6)}$. Se recomienda el uso de antibióticos profilácticos durante la inducción anestésica y continuarlos al menos 24 horas postoperatorias ${ }^{(7)}$.

El uso de $1 \mathrm{mg} / \mathrm{kg}$ al día de prednisona ha demostrado ser eficaz para el aumento de plaquetas de al menos $150,000 / \mu \mathrm{L}$; sin embargo, sólo de 20-30\% de los pacientes presentan remisión prolongada, ésta es definida como aquélla con duración mayor a seis meses. El aumento de plaquetas con el uso de glucocorticoides se ha atribuido por aumento de producción de las mismas, al mismo tiempo que la disminución del secuestro esplénico y la prolongación de la vida media. Será necesario continuar en el período perioperatorio las dosis equivalentes de esteroides por vía intravenosa debido a que la interrupción 
súbita podría causar insuficiencia adrenal; nosotros utilizamos hidrocortisona preoperatoria y dexametasona intraoperatoria. Las dosis equivalentes de $5 \mathrm{mg}$ de prednisona son $20 \mathrm{mg}$ de hidrocortisona y $0.75 \mathrm{mg}$ de dexametasona ${ }^{(8,9)}$.

Es indispensable la corrección de citopenias previo al procedimiento; ya sea mediante transfusión de plaquetas o glóbulos rojos empacados en caso de anemia. El enfoque laparoscópico ha sido el preferido por mucho tiempo, ya que disminuye la morbilidad perioperatoria y la estadía hospitalaria. Es difícil determinar el número exacto de plaquetas para determinar el procedimiento como seguro. Existen informes sobre la realización del procedimiento con trombocitopenia menor a $10,000 / \mu \mathrm{L}$. El momento de transfusión también es debatible debido a que se ha demostrado pocas diferencias entre la transfusión de plaquetas durante la inducción anestésica y durante la ligadura de la arteria esplénica; no obstante, se recomienda la transfusión de plaquetas en el momento de la ligadura de la arteria esplénica en caso de que el paciente presente menos de $10,000 / \mu \mathrm{L}$ de plaquetas. También existe poca evidencia sobre el nivel adecuado de plaquetas para realizar laringoscopía y evitar hemorragia en la vía aérea secundaria a trauma en el momento de la intubación endotraqueal al igual que hemorragia intracraneal. Debido a esto parece razonable transfundir plaquetas a aquellos pacientes que estarán bajo anestesia endotraqueal con trombocitopenia menor a 50,000/ $\mu \mathrm{L}$. A pesar de esto, se ha publicado una serie de casos de pacientes sin complicaciones durante la manipulación de la vía aérea que presentaban menos de $10,000 / \mu \mathrm{L}$ de plaquetas ${ }^{(6,10-12)}$.

El enfoque anestésico para nuestro caso fue bajo anestesia general endotraqueal y optimización del estado general de la paciente. Existe la necesidad de realizar una evaluación preoperatoria adecuada, la cual nos permita determinar una vía aérea difícil anticipada. Se deberá tomar mucha precaución para evitar trauma y sangrado durante la manipulación de la vía aérea. En esta paciente, a pesar de tener múltiples predictores de vía aérea difícil, no se presentó ninguna complicación durante la intubación ${ }^{(7,10,11)}$.

Los eventos trombóticos postoperatorios pueden presentarse hasta en $10 \%$ de los pacientes. Clínicamente pueden presentarse como trombosis portal, esplénica, pulmonar o trombosis venosa profunda. Suelen estar asociados a esplenomegalia y su incidencia no varía mucho entre el abordaje laparoscópico o abierto. Las medias de compresión deberán ser utilizadas por todos los pacientes sometidos a esta cirugía; asimismo, el uso de aspirina en dosis bajas en el postoperatorio podría ser beneficioso si las plaquetas aumentan a más de $800,000 / \mu \mathrm{L}$, al igual que el uso de heparina de forma profiláctica durante el postoperatorio. Sin embargo, no existen protocolos establecidos para evitar esta complicación $^{(7,13)}$.

\section{CONCLUSIONES}

Este caso, al igual que algunos similares en la bibliografía, hace referencia sobre la importancia de una valoración preanestésica adecuada; se enfatiza en la optimización del paciente previo a la cirugía, evitar el trauma durante la manipulación de la vía aérea y el uso de esteroides durante el período prey postoperatorio. A pesar de que existen publicaciones de cirugías sin complicaciones con trombocitopenia severa, y por tratarse de una cirugía electiva, se prefiere la transfusión previa de plaquetas para disminuir la morbilidad y mortalidad perioperatoria.

\section{REFERENCIAS}

1. Hansen DL, Moller S, Andersen K, et al. Evans syndrome in adults - incidence, prevalence, and survival in a nationwide cohort. Am J Hematol. 2019;94:1081-1090.

2. Al Hazmi A, Winters M. Evans Syndrome. Clinical Practice and Cases in Emergency Medicine. 2019;2:128-131.

3. Jaime-Perez JC, Aguilar-Calderon P, Cavazos L, et al. Evans Syndrome: clinical perspectives, biological insights and treatment modalities. Journal of Blood Medicine. 2018;9:171-184.

4. Jaime-Perez JC, Guerra-Leal LN, Lopez-Razo ON, et al. Experience with Evans Syndrome in an academic referral center. Brazilian Journal of Hematology and Hemotherapy. 2015;37:230-235.

5. Monga V, Maliske S, Perepu U. Fatal pulmonary embolism following splenectomy in a patient with Evans syndrome: case report and review of the literature. Thrombosis Journal. 2017;15:1-10.

6. Bonnet S, Guedon A, Ribeil J-A, et al. Indications and outcomes of splenectomy in hematologic diseases. Journal of Visceral Surgery. 2017;154:421-429.

7. Moris D, Dimitriou N, Griniatsos J. Laparoscopic Splenectomy for Benign Hematological Disorders in Adults: A Systematic Review. In Vivo. 2017;31:291-302.
8. Houwerzijl EJ, Louwes H, Sluiter WJ, et al. Platelet production rate predicts the response to prednisone therapy in patients with idiopathic thrombocytopenic purpura. Ann Hematol. 2008;87:975-983.

9. Liu, Dora et al. A practical guide to the monitoring and management of the complications of systemic corticosteroid therapy. Allergy, asthma, and clinical immunology. 2013;9:1-30.

10. Jonnavithula N, Pasupulenti S, Thumma V, et al. Anesthetic management of laparoscopic splenectomy in a case of Evans syndrome with systemic lupus erythematosis. Journal of Anesthesiology Clinical Pharmacology. 2018;34:263-264.

11. Duperier T, Felsher J, Brody F. Laparoscopic splenectomy for Evans syndrome. Surgical Laparoscopy Endoscopy Percutaneous Techniques. 2003;13:45-47.

12. Gupta S, Kalayarsen R, Chandrasekar S, et al. Laparoscopic Splenectomy for Inmune Thrombocytopenic Purpura (ITP) Patients with Very Severe Thrombocytopenia. Indian Journal of Hematology and Blood Transfusion. 2018;34:535-539.

13. Weledji EP. Benefits and risks of splenectomy. International Journal of Surgery. 2014;12:113-9. 